\title{
Pulmonary arteriovenous malformations in children with palliated univentricular CHD: our limited understanding persists
}

\section{Letter to the Editor}

Cite this article: Spearman AD and Ginde S (2020) Pulmonary arteriovenous malformations in children with palliated univentricular CHD: our limited understanding persists. Cardiology in the Young 30: 597-598. doi: 10.1017/S1047951120000736

Received: 12 February 2020 Accepted: 27 February 2020 First published online: 6 April 2020

Author for correspondence: A. D. Spearman, Department of Pediatrics, Division of Cardiology, Medical College of Wisconsin, Children's Wisconsin, Herma Heart Institute, 9000 West Wisconsin Avenue, Milwaukee, WI 53226, USA. Tel: +414 266 2000; Fax: +414 266 3261;

E-mail: aspearman@mcw.edu (c) The Author(s) 2020. Published by Cambridge University Press.

\section{Andrew D. Spearman ${ }^{1,2}$ (1) and Salil Ginde $\mathrm{e}^{1,2,3}$}

${ }^{1}$ Department of Pediatrics, Division of Cardiology, Medical College of Wisconsin, Children's Wisconsin, Herma Heart Institute, Milwaukee, WI, USA; ${ }^{2}$ Cardiovascular Center, Medical College of Wisconsin, Milwaukee, WI, USA and ${ }^{3}$ Department of Medicine, Division of Cardiology, Medical College of Wisconsin, Milwaukee, WI, USA

Dear Editor,

We were excited to read the manuscript by Asada et al. investigating bubble contrast echocardiography in children after total cavopulmonary connection. ${ }^{1}$ Asada et al. retrospectively analysed the medical records of patients who underwent cardiac catheterisations and bubble contrast (agitated saline) transthoracic echocardiograms from 2010 to 2018 . Their study impressively included 140 patients (mean age 7.6 [range 2.5-20.8] years) who previously underwent total cavopulmonary connection (mean of 5.3 [range $0.6-18.1$ ] years after total cavopulmonary connection). They reported that only 13 patients (9.3\%) had negative bubble studies bilaterally and that there was no correlation between bubble study grade and pulmonary capillary wedge saturation (a surrogate for pulmonary vein oxygen saturation). They concluded that bubble contrast echocardiography may create false positive findings for detecting pulmonary arteriovenous fistulas and further standardisation of bubble echocardiograms is required.

We advocate for more conservative interpretation of their findings. Asada et al. reported that bubble contrast grade did not correlate with pulmonary capillary wedge saturation, but this was well demonstrated more than 20 years ago. ${ }^{2,3}$ Feinstein et al. rationalised that bubble echocardiography is a sensitive test capable of identifying pulmonary microvascular disease before pulmonary vein desaturation. They recommended improving echo correlation by assessing the partial pressure of oxygen $\left(\mathrm{PaO}_{2}\right)$ in pulmonary veins while patients inspire $100 \% \mathrm{O}_{2}$. Abnormally low $\mathrm{PaO}_{2}(<500 \mathrm{mmHg})$ on supplemental oxygen indicates impaired gas exchange in the pulmonary capillaries despite normal saturation and has a negative predictive value of $100 \%{ }^{4}$ Recent guidelines for assessing pulmonary arteriovenous malformations in patients with hereditary haemorrhagic telangiectasia support the use of transthoracic bubble contrast echocardiography as the initial screening test given its high sensitivity, and a positive bubble study without other abnormalities is diagnostic of microscopic pulmonary arteriovenous malformations. ${ }^{5}$ Importantly, it is unknown if or how pulmonary arteriovenous malformations differ between patients with univentricular CHD and patients with hereditary haemorrhagic telangiectasia.

Regardless of bubble contrast correlation with oxygen saturation or $\mathrm{PaO}_{2}$, it is unknown if gradually improved oxygen saturations that are normally observed after total cavopulmonary connection indicate pulmonary vascular reverse remodelling with complete microscopic resolution of pulmonary arteriovenous malformations or simply functional improvement in gas exchange. ${ }^{6,7}$ A positive bubble study 5 years after total cavopulmonary connection with normal oxygen saturation may indicate functional resolution of pulmonary arteriovenous malformations without complete microvascular remodelling. In this scenario, a positive bubble study is not a false positive but rather a sensitive biomarker of subclinical pulmonary microvascular disease. In other words, once pulmonary arteriovenous malformations are established, they may never fully remodel to normal pulmonary microvasculature.

Asada et al. assert that the efficacy of bubble echocardiography for the diagnosis of pulmonary arteriovenous malformations has not been properly evaluated and standardisation of the technique is required. We agree in principal with their concerns about bubble echocardiography assessing pulmonary arteriovenous malformations in patients with univentricular CHD; however, we propose that the main limitation falls on our poor understanding of pulmonary arteriovenous malformations and pulmonary vascular remodelling in patients with palliated univentricular CHD.

Acknowledgements. None.

Financial support. This research received no specific grant from any funding agency, commercial or not-forprofit sectors.

Conflicts of interest. None. 


\section{References}

1. Asada D, Morishita Y, Kawai Y, Kajiyama Y, Ikeda K. Efficacy of bubble contrast echocardiography in detecting pulmonary arteriovenous fistulas in children with univentricular heart after total cavopulmonary connection. Cardiol Young 2020 Jan 9, 1-4. doi: 10.1017/S104795111900324X.

2. Chang RK, Alejos JC, Atkinson DJ, et al. Bubble contrast echocardiography in detecting pulmonary arteriovenous shunting in children with univentricular heart after cavopulmonary anastomosis. J Am Coll Cardiol 1999; 33: 2052-2058.

3. Feinstein JA, Moore P, Rosenthal DN, Puchalski M, Brook MM. Comparison of contrast echocardiography versus cardiac catheterization for detection of pulmonary arteriovenous malformations. Am J Cardiol 2002; 89: 281-285.
4. Kjeldsen AD, Oxhoj $\mathrm{H}$, Andersen PE, et al. Pulmonary arteriovenous malformations: screening procedures and pulmonary angiography in patients with hereditary hemorrhagic telangiectasia. Chest 1999; 116: 432-439.

5. Faughnan ME, Palda VA, Garcia-Tsao G, et al. International guidelines for the diagnosis and management of hereditary haemorrhagic telangiectasia. J Med Genet 2011; 48: 73-87.

6. McElhinney DB, Kreutzer J, Lang P, Mayer JE Jr, del Nido PJ, Lock JE. Incorporation of the hepatic veins into the cavopulmonary circulation in patients with heterotaxy and pulmonary arteriovenous malformations after a Kawashima procedure. Ann Thorac Surg 2005; 80: 1597-1603.

7. Kutty S, Frommelt MA, Danford DA, Tweddel JS. Medium-term outcomes of Kawashima and completion Fontan palliation in single-ventricle heart disease with heterotaxy and interrupted inferior vena cava. Ann Thorac Surg 2010; 90: 1609-1614. 